\title{
Development of a water-cooled multi-hole calibration burner for optical measurements of flames with high pressures and temperatures
}

\author{
Kiyonori TAKEUCHI*, Yoshio NUNOME**, Sadatake TOMIOKA**, Takeo TOMITA**, Taku KUDO*, \\ Akihiro HAYAKAWA* and Hideaki KOBAYASHI* \\ *Institute of Fluid Science, Tohoku University \\ 2-1-1 Katahira, Aoba-ku, Sendai, Miyagi 980-8577, Japan \\ E-mail: takeuchi@flame.ifs.tohoku.ac.jp \\ ** Research and Development Directorate, Japan Aerospace Exploration Agency \\ 1 Koganezawa, Kimigaya-aza, Kakuda, Miyagi 981-1525, Japan
}

Received: 19 September 2017; Revised: 28 November 2017; Accepted: 19 December 2017

\begin{abstract}
High temperature flames, which can be produced via oxygen-enriched combustion, potentially have improved the combustion characteristics over air-only combustion flames due to their substantially higher flame temperatures. These conditions necessitate the use of non-intrusive optical measuring methods to measure the temperature and the chemical species in the flame. To develop an optical measurement calibration burner that can be used at high pressure and temperature conditions, a new calibration burner which employed water-cooled multi-hole nozzle was developed in this study. Premixed $\mathrm{CH}_{4} / \mathrm{O}_{2} / \mathrm{N}_{2}$ oxygen-enriched conditions were selected to investigate both the heat-resisting properties of the developed burner nozzle and the burner's flame characteristics. OH-Planar Laser-Induced Fluorescence (OH-PLIF) measurements were conducted on the flames to observe the $\mathrm{OH}$ distributions. The flame temperature at $0.10 \mathrm{MPa}$ was derived using a Boltzmann-plot for the $\mathrm{OH}$ fluorescence excitations. To verify the variation of molecular concentration with equivalence ratio for the experimental flames qualitatively, the experimentally acquired $\mathrm{OH}$ and $\mathrm{CH}$ chemiluminescence intensities were compared with the simulated partial pressure of $\mathrm{OH}^{*}$ and $\mathrm{CH}^{*}$, respectively. Experimental results showed that the $\mathrm{CH}_{4} / \mathrm{O}_{2} / \mathrm{N}_{2}$ flames were stabilized on the burner nozzle in a wide range of oxygen-enrichment ratio, from 0.40 to 1.0 at atmospheric pressure. At an oxygen-enrichment ratio of 0.45 , the flames were also stabilized in pressure conditions up to $0.49 \mathrm{MPa}$, while the inner nozzle temperature was lower than $400 \mathrm{~K}$. The OH-PLIF images showed that the $\mathrm{OH}$ was distributed almost uniformly along the axial direction of the burner, and demonstrated similar characteristics to that of a flat flame. The derived maximum flame temperature at atmospheric pressure was approximately $2650 \mathrm{~K}$ at an oxygen-enrichment ratio of 0.80 . The variation of the $\mathrm{OH}$ and $\mathrm{CH}$ chemiluminescence intensities with change of equivalence ratios corresponded roughly with the simulated partial pressures of $\mathrm{OH}^{*}$ and $\mathrm{CH}^{*}$ at each pressure condition.
\end{abstract}

Keywords : Oxygen-enriched flames, Burner design, High pressure combustion, Laser induced fluorescence, Chemiluminescence

\section{Introduction}

Pure oxygen combustion and oxygen enriched combustion are techniques that are widely used in application such as liquid bi-propellant rocket engines and industrial burners. Oxygen-enriched combustion has garnered significant attention due to its high calorific value and burning velocity which contributes to a reduction of exhaust gas (Nishimura et al., 2000, Shi et al., 2012), stabilization of mildly flammable fuels (Takeishi et al., 2015), high energy gains, and potential applications to low energy fuel combustion. The most notable characteristic of oxygen-enriched combustion is its high flame temperature. Compared with air-only combustion, an oxygen-enriched condition has substantially increased energy 
efficiencies. Additionally, when methane is used for the fuel, the adiabatic flame temperature can reach as high as 2700 $\mathrm{K}$ under atmospheric pressure (Shi et al., 2012). In pure oxygen combustion, there are many fuels with adiabatic flame temperatures as high as $3000 \mathrm{~K}$ at maximum (Niioka et al., 2000).

This increased flame temperature creates problems for combustion measurement. High flame temperatures necessitate the use of non-intrusive optical measuring methods based on spectroscopy to measure the temperature and the chemical species in the flame. When an optical measurement is selected, which represents such non-intrusive measurement method, a calibration must be conducted for the method, because spectroscopic measurement handles spectroscopic variables that feature strong temperature and pressure dependences. To date, various non-intrusive measuring methods have been developed and applied for studies of combustion diagnostics. Moreover, two-dimensional combustion measuring methods such as Planar Laser-Induced Fluorescence (PLIF) have been also used frequently in recent years. Additionally, when a new combustion measurement technique is devised, it must be verified with results to confirm its accuracy. The most general method for calibrating a combustion measurement is to use a calibration burner, which creates a quasi-one-dimensional combustion field. Thus, a calibration burner is used in many combustion diagnostic studies.

There are many types of calibration burners, but the essential concept of all types is to create a uniform combustion field in the radial direction to assure one-dimensional properties of the field. There are two common methods to create this uniform field: use a porous material such as sintered metal or make many small holes or tubes as the shape of the exit for the unburnt gas. So-called McKenna burner (Holthuis \& Associates) is a widely used calibration burner model that uses sintered metal, and experimental data, such as temperature measurements, have been reported via Coherent Anti-Stokes Raman Spectroscopy (CARS) at each combustion condition (Weigand et al., 2017, Prucker et al., 1994). It has been reported that it is possible to stabilize a stable laminar flat flame up to 60 bar via heat loss to the surface of the sintered metal (Bessler et al., 2002, Cheng et al., 2002). However, stabilizing a flat flame by heat loss causes a strong heat load on the sintered metal; thus, for conditions in which the flame temperature is higher than the temperature in above case, another burner type should be used according to Kojima et al(2003). Meanwhile, a nozzle shape with many small holes or tubes such as a Hencken burner and Meker burner must keep the injection velocity of the premixed gas faster than the burning velocity when the flame quenching diameter is smaller than the diameter of the burner exit. However, the heat load on the injection surface of the burners is estimated to be lower than sintered metal. As an example, stabilization of hydrogen-air rapid premixed flame at $10 \mathrm{~atm}$, has been reported using a nozzle with non-cooling multiple small holes, but its gas outflow surface reached temperatures as high as $700 \mathrm{~K}$ (Kojima et al., 2003). Although there are many reports on calibration burners successfully operating in high pressure conditions, reports on their operation in oxygen-enriched conditions with high flame temperatures are scarce. The heat resistance of these burner is a cause of this problem. It is an urgent task to develop a calibration burner that can accurately realize a relatively wide quasi-onedimensional uniform temperature and species field with actual flame temperatures associated with oxygen-enriched or pure oxygen combustion.

In this study, a new optical measurement calibration burner is presented that can be used for calibration and quantification of high flame temperatures such as oxygen-enriched flames in pressures ranging from atmospheric to highest pressure condition of $0.49 \mathrm{MPa}$. Oxygen-enriched methane/oxygen/nitrogen $\left(\mathrm{CH}_{4} / \mathrm{O}_{2} / \mathrm{N}_{2}\right)$ flames were formed with maximum chamber pressure up to $0.49 \mathrm{MPa}$, and the basic performance of the burner (e.g., flame stabilities and heat resistance of the nozzle) was verified. The emission distribution of $\mathrm{OH}$ radicals was acquired by an $\mathrm{OH}-\mathrm{Planar}$ Laser-induced Fluorescence (OH-PLIF) measurement to examine the uniformity of $\mathrm{OH}$ distribution in region of downstream of the nozzle. Furthermore, a numerical simulation of the one-dimensional flame as well as an experiment using the Boltzmann plot method were conducted to examine the flame temperature. Moreover, the simulated partial pressure of excited chemical species $\mathrm{OH}^{*}$ and $\mathrm{CH}^{*}$ were compared with the corresponding $\mathrm{OH}$ and $\mathrm{CH}$ chemiluminescence intensities which were acquired in the experiments to verify the variation of molecular concentration with equivalence ratio for the experimental flames qualitatively.

\section{Experimental and numerical method}

\subsection{Newly developed water-cooled multi-hole calibration burner}

The newly-devised calibration burner designed and developed in this study is shown in Fig. 1. This burner was designed to be cooled by water considering its high heat load conditions, in contrast to so-called Hencken burner. The 


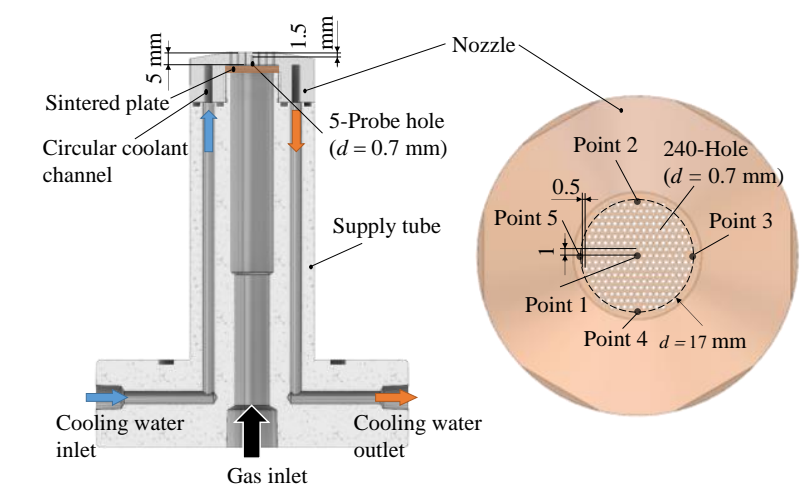

Fig. 1 Schematic diagram of calibration burner using water-cooled multi-hole nozzle designed in this study.

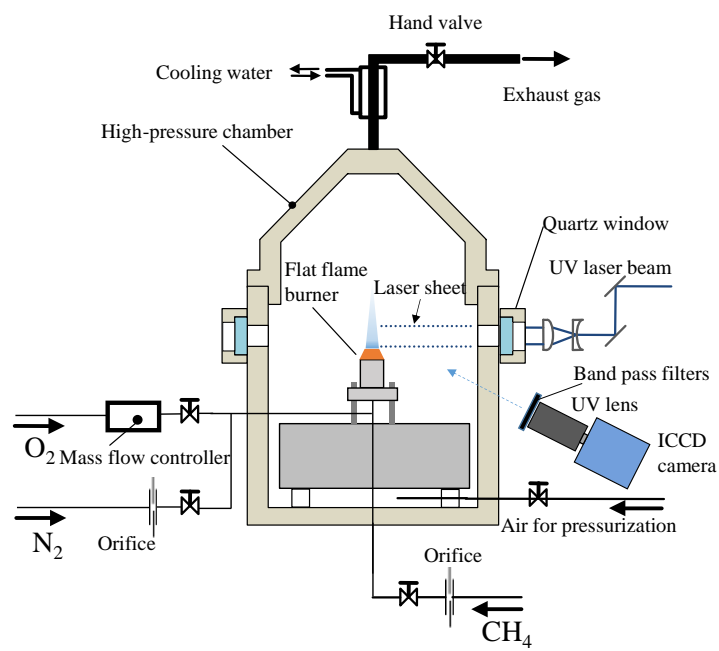

Fig. 2 Schematic of high pressure combustion test facility.

burner was composed of only two parts: a nozzle made from oxygen-free copper, which has a high heat conductivity, and a supply tube made of stainless steel. Parallel pipe thread connected the nozzle and supply tube. The nozzle was so designed that it was easy to detach considering the increasing damage for the nozzle with these operating conditions. The center of the supply tube was used for supplying the premixed gas and there were four channels around the outer perimeter through which the cooling water could flow. This experiment used water for the coolant and the temperature and flow rate were approximately $293 \mathrm{~K}$ and $11 \mathrm{~L} / \mathrm{min}$, respectively. The bottom of the supply tube had a flange shape to match the interface with the high pressure combustion chamber (Takeuchi et al., 2017). The nozzle surface attached to the supply tube had a $3 \mathrm{~mm}$ wide circular coolant channel for flow on the outer perimeter. On the upper nozzle surface, 240 small holes with a diameter of $0.7 \mathrm{~mm}$ in a staggered arrangement were provided to fit inside a $17 \mathrm{~mm}$ diameter circumference to assure the formation of one-dimensional flame with the same way as so-called Hencken burner. The length of the holes was $5 \mathrm{~mm}$, and the aspect ratio of the holes was 7.14. Furthermore, four insertion holes for a thermocouple were placed every 90 degrees around the $17 \mathrm{~mm}$ diameter as well as a single point in the center; thus, there were a total of five points to measure the radial temperature distribution of the injection surface during flame formation. The center hole for the thermocouple was named Point1, and two holes along the $17 \mathrm{~mm}$ diameter were named Point 2 and Point3, as shown in Fig. 1. There was a $3 \mathrm{~mm}$ clearance between the nozzle and supply tube to insert sintered metal to prevent flame oscillation, flashback and to enhance mixing as well as so-called McKenna burner. By adopting these features, the burner can operate in the high pressure and temperature conditions.

\subsection{Procedure of high pressure combustion and optical measurements}

Combustion experiments were carried out using the high pressure combustion test facility in the Institute of Fluid Science, Tohoku University. As shown in Fig. 2, the burner was placed inside the chamber with a chamber pressure $P_{\mathrm{c}}=0.10 \sim 0.49 \mathrm{MPa}$ along with $\mathrm{OH}-\mathrm{PLIF}$ measurement. The orifice or mass flow controller were used to measure each of the $\mathrm{CH}_{4} / \mathrm{O}_{2} / \mathrm{N}_{2}$ gas flow rates. Compressed air was used to pressurize the chamber. The average premixed gas flow velocity of each hole was set to be above the laminar burning velocity. The largest Reynolds number for each hole was 
approximately 1250 for $P_{c}=0.49 \mathrm{MPa}$ and an equivalence ratio $\phi=1.2$. Only the $\mathrm{CH}_{4}$ flow rate was changed to adjust the equivalence ratio. Each gas flow rate was determined by the oxygen-enrichment ratio $\beta$ and the equivalence ratio $\phi$. By using volumetric flow rate $Q$ of $\mathrm{O}_{2}$ and $\mathrm{N}_{2}, \beta$ is defined as given Eq. (1).

$$
\beta=\frac{Q_{O 2}}{Q_{O 2}+Q_{N 2}}
$$

According to this equation, $\beta=0.21$ corresponds to air. In this study, the combustion experiment was carried out with $\beta$ $=0.40 \sim 1.0$ at atmospheric pressure and $\beta=0.45$ at high pressure.

The OH-PLIF measurement was conducted to examine the flame formed above the burner as well as downstream of the flame. A Nd:YAG laser with an oscillation frequency of $10 \mathrm{~Hz}$ and a dye laser were used for the light sources to excite the $\mathrm{OH}$ in $\mathrm{OH}-\mathrm{PLIF}$ measurement. The laser beam power was approximately $14 \mathrm{~mJ} / \mathrm{pulse}$ at the dye laser outlet. The laser beam was formed into a sheet $40 \mathrm{~mm}$ in height above the nozzle surface and $0.1 \mathrm{~mm}$ in thickness using cylindrical silica lenses. The $\mathrm{OH}(2,0)$ band $\mathrm{Q}_{1}(9)$ branch $(263.580 \mathrm{~nm})$ was selected as the excitation line due to the relatively small temperature dependence of $f_{\mathrm{B}} / T$ for the predicted flame temperature range of $2500 \sim 3000 \mathrm{~K}$. The temperature dependence of $f_{\mathrm{B}} / T$ is given in Eq. 2 (Sakiyama et al., 1999).

$$
\frac{f_{B}}{T}=\frac{2 J^{\prime \prime}+1}{T} \exp \left\{-B_{V} J^{\prime \prime}\left(J^{\prime \prime}+1\right) /(k T)\right\}
$$

Here, $f_{\mathrm{B}}$ is the Boltzmann factor, $T$ is the temperature $[\mathrm{K}], J$ " is the total angular momentum quantum number of the vibrational ground state, $B_{V}$ is the rotational constant $=18.513 \mathrm{~cm}^{-1}$ at the vibrational ground state, and $k$ is the Boltzmann constant. The variation range of $f_{\mathrm{B}} / T$ of the $\mathrm{Q}_{1}(9)$ branch is $0.1 \%$ in the temperature range of $2500 \sim 3000 \mathrm{~K}$.

The photon detector system is composed of an ICCD camera (Andor, USB-iStar), an ultraviolet lens (Nikon, UV-105 mm F4.5), and optical filters. The frame rate of the ICCD is approximately $4 \mathrm{~Hz}$, and the gate duration of the image intensifier is approximately $25 \mathrm{~ns}$. $\mathrm{OH}(2,1)$ band fluorescence was acquired by using a combined optical filter consisting of a color glass filter UG11 (SCHOTT, $325 \mathrm{~nm}$ centerd, FWHM of $110 \mathrm{~nm}$ ) and an interference filter HQBP290-UV (Asahi spectra, $290 \mathrm{~nm}$ centered, $10 \mathrm{~nm}$ of FWHM).

Temperature measurements using the Boltzmann plot advocated by Anderson et al (1982) were conducted in the combustion condition with a chamber pressure $P_{\mathrm{C}}=0.10 \mathrm{MPa}$ and $\beta=0.2,0.4$, and 0.6 . This measurement technique is the standard method for obtaining the temperature from the slope by plotting each rotational and vibrational state of the molecule measured by Laser-Induced Fluorescence (LIF) to ground state of rotational energy $\omega^{\prime \prime}=B \vee J(J+1)\left[\mathrm{cm}^{-1}\right]$. The relationship between fluorescence intensity $S_{\mathrm{FL}}$ and rotational energy $\omega$ "' is shown in Eq. (3) (Anderson et al., 1982).

Table 1 Details of $\mathrm{OH}(2,0)$ band excitation lines.

\begin{tabular}{cccc}
\hline Branch & $\begin{array}{c}\text { Total angular momentum } \\
\text { quantum number } J^{\prime \prime}\end{array}$ & $\begin{array}{c}\text { Wavelength } \\
\lambda[\mathrm{nm}]\end{array}$ & $\begin{array}{c}\text { Grond-State Energy } \\
\omega^{\prime \prime}[\mathrm{cm}-1]\end{array}$ \\
\hline Q1(1) & 1.5 & 261.346 & 69.431 \\
Q1(2) & 2.5 & 261.507 & 162.006 \\
Q1(3) & 3.5 & 261.685 & 291.611 \\
Q1(4) & 4.5 & 261.902 & 458.246 \\
Q1(5) & 5.5 & 262.156 & 661.911 \\
Q1(6) & 6.5 & 262.449 & 902.606 \\
Q1(7) & 7.5 & 262.784 & 1180.331 \\
Q1(8) & 8.5 & 263.163 & 1495.086 \\
Q1(9) & 9.5 & 263.586 & 1846.871 \\
Q1(10) & 10.5 & 264.055 & 2235.686 \\
Q1(11) & 11.5 & 264.571 & 2661.531 \\
Q1(12) & 12.5 & 265.135 & 3124.406 \\
Q1(13) & 13.5 & 265.750 & 3624.311 \\
\hline
\end{tabular}




$$
\ln \left\{\frac{S_{\mathrm{FL}}}{B_{12}\left(2 J^{\prime \prime}+1\right)}\right\}=-\frac{\omega^{\prime \prime}}{k T}+C
$$

Here, $B_{12}$ is the stimulated absorption coefficient (Einstein's $B$ coefficient) $\left[\mathrm{m}^{2} /(\mathrm{J} \cdot \mathrm{s})\right]$ ) which was acquired by using the reference from spectroscopic simulation software LIFBASE (2017). $C$ is the arbitrary slope constant derived from the Boltzmann plot. In this study, the fluorescence intensity $S_{\mathrm{FL}}$ of each $\mathrm{OH}(2,0)$ band $\mathrm{Q}_{1}$ branch rotational state was measured via the OH-PLIF equipment as explained above. The total angular momentum quantum number at ground state $J$ ', the wavelength $\lambda$ and the rotational energy at ground state $\omega$ " are shown in Table 1.

The flame temperature and the concentration field of the chemical species were also evaluated by measuring the chemiluminescence intensities of chemical species that have strong temperature dependences and by comparing them with simulated partial pressures of the chemical species. $\mathrm{OH}$ and $\mathrm{CH}$ were selected as the chemical species to be measured in this study. The same ICCD camera from the PLIF measurements was used to detect the chemiluminescence intensity. A filter combination of UG11 and HQBP310-UV (Asahi spectra, $310 \mathrm{~nm}$ centered, $10 \mathrm{~nm}$ of FWHM) was used to detect $\mathrm{OH}(0,0)$ band chemiluminescence, and a combination of UG11 and an interference filter (Optical Coating Japan, $432 \mathrm{~nm}$ centered, $17.4 \mathrm{~nm}$ of FWHM) was used to detect the $\mathrm{CH}(0,0)$ band chemiluminescence.

\subsection{One-dimensional laminar flame numerical simulation}

In addition to the experiment, the laminar flame velocity, mole fraction of each chemical species, and flame temperature at each combustion condition were evaluated by using a commercial reaction analysis software CHEMKINPro (2017) with the Premixed Laminar Flame-Speed model. A CH $4 / \mathrm{DME}$ (Dimethyl ether) model composed of 113 chemical species and 710 elementary reactions (Burke et al., 2015) was used for the reaction kinetics. The radiation heat loss was modeled using Planck mean absorption coefficients [Barlow et al., 2001]. In this study, the unburnt premixed gas temperature and ambient temperature were considered to be $293 \mathrm{~K}$ by disregarding the heat loss to the burner. The excited chemical species of $\mathrm{OH}^{*}$ and $\mathrm{CH}^{*}$ were included to the reaction mechanism and their partial pressures were compared with the chemiluminescence intensities of the $\mathrm{OH}(0,0)$ band and $\mathrm{CH}(0,0)$ band obtained by the experiments.

\section{Results and discussion 3.1 Heat resistance of burner nozzle}

The oxygen-enrichment ratio and chamber pressure conditions were restricted by the heat resistance of the burner nozzle. The finite-element analysis conducted during the nozzle design stage indicated that the safety factor becomes less than 1 when the nozzle surface temperature reaches approximately $470 \mathrm{~K}$. To ensure the safe operation of the burner, the inner nozzle temperature was evaluated. The variations of the inner nozzle temperature in Point 1 with equivalence ratio and net heat production rate at each pressure condition are shown in Fig. 3. The net heat production was calculated by

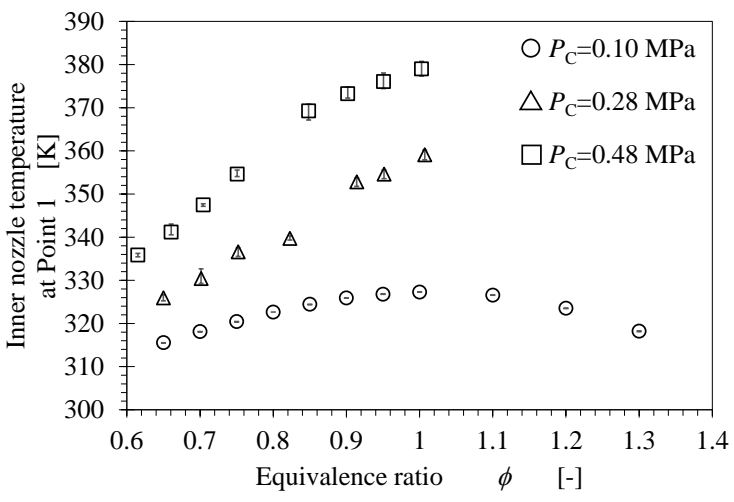

(a) Dependence of equivalence ratio

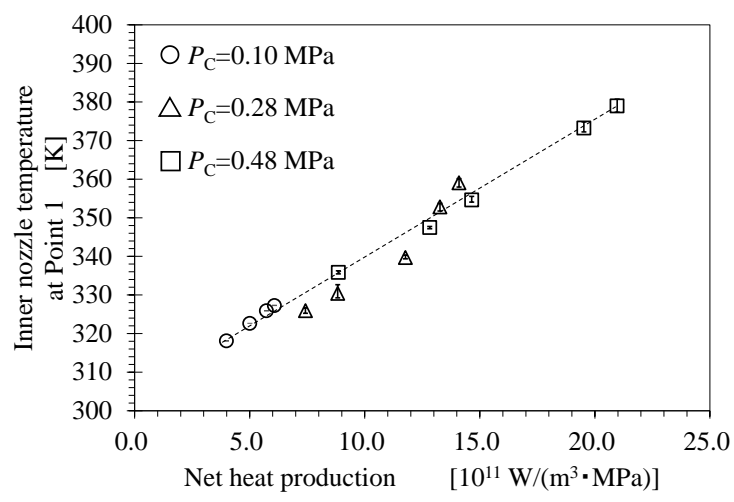

(b) Dependence of peak of net heat production

Fig. 3 Inner nozzle temperature measured at each pressure $P_{\mathrm{C}}$. The temperatures measured at $P_{\mathrm{C}}=0.10,028$ and $0.49 \mathrm{MPa}$ are indicated by circle, triangles and squares, respectively. The oxygen-enrichment ratio $\beta$ is 0.45 . 
the one-dimensional simulation and normalized by the chamber pressure. As shown in Fig. 3(a), both the inner nozzle temperature and the flame temperature had a peak value at approximately $\phi=1.0$. The sintered metal was not inserted when the thermocouple was inserted, and the measurements at fuel enriched regions were not conducted at pressures of $P_{\mathrm{C}}=0.28$ and $0.48 \mathrm{MPa}$ because a loud vibration sound was released in the audible range, which was assumed to be flame oscillation. Numerical simulations also indicated that the nozzle inner temperature was around $380 \mathrm{~K}$ and the flame temperature was approximately $2900 \mathrm{~K}$ at the highest heat load condition in this study with $P_{\mathrm{C}}=0.48$ $\mathrm{MPa}, \beta=0.45$, and $\phi=1.0$ in this study. It can be concluded that the nozzle has a strong cooling performance and appropriate heat resistance for the experimental conditions. Moreover, the nozzle surface could be treated as an isothermal wall because the nozzle inner temperature history was almost steady at all flame conditions. From Fig. 3(b), it can be observed that there is a linear relationship between the inner temperature of the nozzle and the net heat production normalized by chamber pressure. From the linear relationship, the inner temperature of the nozzle at a certain pressure condition can be estimated by calculating the net heat production when the coolant temperature and flow velocity are constant.

\subsection{Results of OH-PLIF measurements}

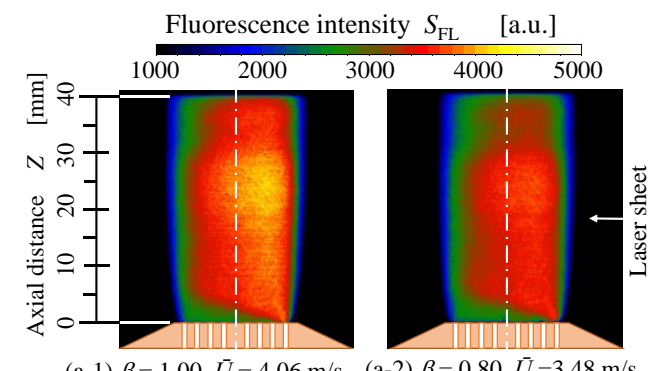

(a-1) $\beta=1.00, \bar{U}=4.06 \mathrm{~m} / \mathrm{s} \quad(\mathrm{a}-2) \beta=0.80, \bar{U}=3.48 \mathrm{~m} / \mathrm{s}$

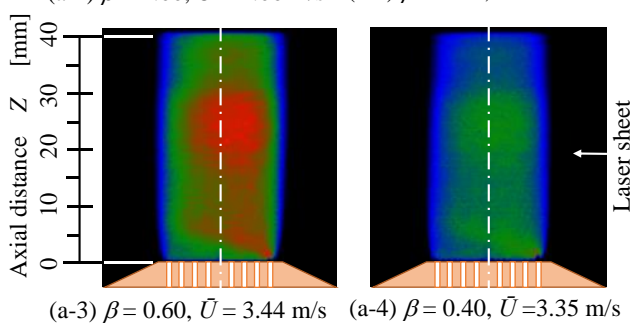

(a) $P_{\mathrm{C}}=0.10 \mathrm{MPa}, \phi=1.0$

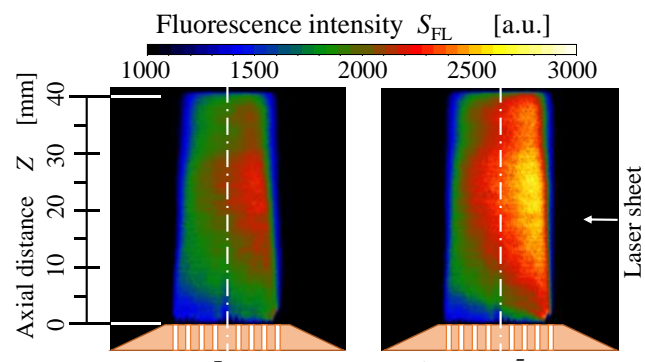

(c-1) $\phi=0.60, \bar{U}=4.83 \mathrm{~m} / \mathrm{s} \quad$ (c-2) $\phi=0.80, \bar{U}=4.82 \mathrm{~m} / \mathrm{s}$

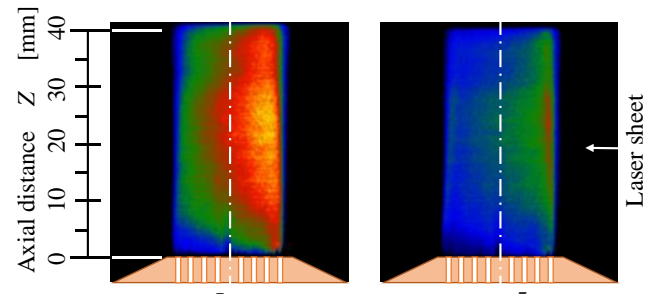

(c-3) $\phi=1.00, \bar{U}=5.13 \mathrm{~m} / \mathrm{s} \quad$ (c-4) $\phi=1.20, \bar{U}=5.20 \mathrm{~m} / \mathrm{s}$

(c) $P_{\mathrm{C}}=0.29 \mathrm{MPa}, \beta=0.45$

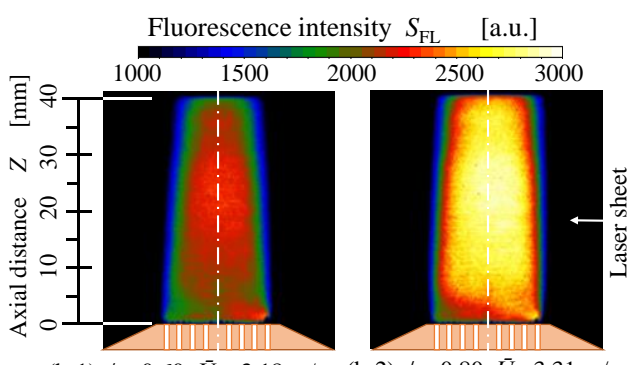

(b-1) $\phi=0.60, \bar{U}=3.18 \mathrm{~m} / \mathrm{s} \quad(\mathrm{b}-2) \phi=0.80, \bar{U}=3.31 \mathrm{~m} / \mathrm{s}$

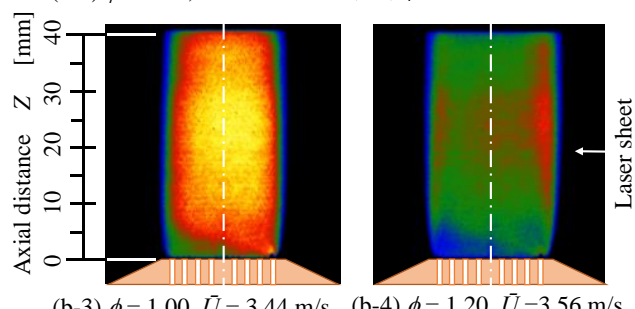

(b-3) $\phi=1.00, \bar{U}=3.44 \mathrm{~m} / \mathrm{s} \quad$ (b-4) $\phi=1.20, \bar{U}=3.56 \mathrm{~m} / \mathrm{s}$

(b) $P_{\mathrm{C}}=0.10 \mathrm{MPa}, \beta=0.45$

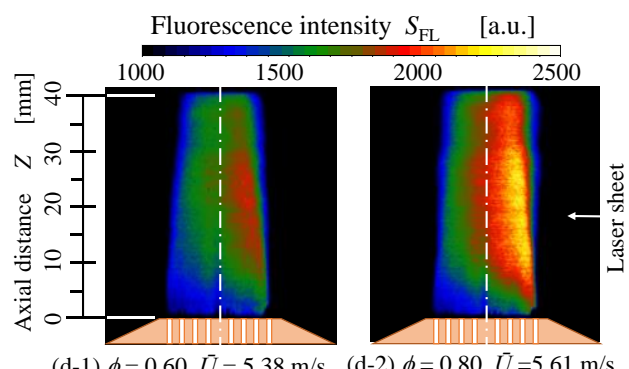

(d-1) $\phi=0.60, \bar{U}=5.38 \mathrm{~m} / \mathrm{s} \quad(\mathrm{d}-2) \phi=0.80, \bar{U}=5.61 \mathrm{~m} / \mathrm{s}$

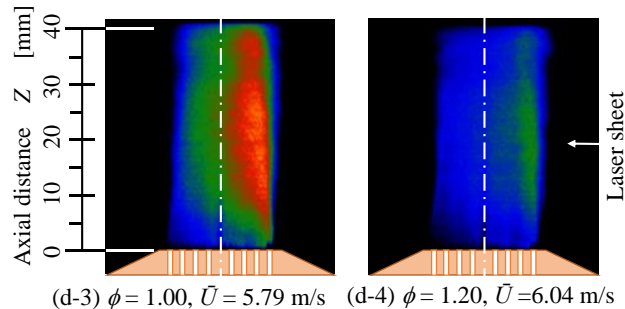

(d) $P_{\mathrm{C}}=0.49 \mathrm{MPa}, \beta=0.45$

Fig. 4 Multi-shot-averaged OH-PLIF images obtained from the output of image processing 100 single-shot images.

(a) shows the variation of oxygen-enrichment ratio $\beta$ at $P_{\mathrm{C}}=0.10 \mathrm{MPa}$ and $\phi=1.0$. (b), (c), and (d) show the variation of the equivalence ratio $\phi$ at $P_{\mathrm{C}}=0.10 \mathrm{MPa}, 0.29$ and $0.49 \mathrm{MPa}$, respectively. 
The averaged $\mathrm{OH}-\mathrm{PLIF}$ images acquired after processing 100 single-shot images for each flame condition are shown in Fig. 4. Figure 4 (a) shows the averaged OH-PLIF image with the conditions $P_{\mathrm{C}}=0.10 \mathrm{MPa}, \phi=1.0$ and $\beta=1.0,0.80$, 0.60 , and 0.40 . A steady flame was stabilized on the nozzle surface for all $\beta$ conditions. It was also observed that the flame consisted of small conical flames on the outlet holes of the premixed gas instead of a flat flame. Small bumps were observed on the nozzle surface when $\beta=0.40$ where there was a big difference between the laminar burning velocity and the average premixed gas flow velocity $\bar{U}$. Meanwhile, attenuation of the fluorescence signal intensity was observed on the right side of the burner axial center which is the side of the laser sheet insertion. This attenuation was more noticeable for highly oxygen enriched conditions such as $\beta=1.0,0.80$, and 0.60 . This is clearly caused by laser absorption becoming stronger when the $\mathrm{OH}$ concentration is high in oxygen enriched flames. Even for the $\mathrm{OH}(2,0)$ band excitation where the absorption intensity is weaker than the general $\mathrm{OH}(1,0)$ band excitation at atmospheric pressure, this effect should not be ignored and method such as Beer-Lambert's law (Daily, 1997) must be used to correct the laser-power degradation from $\mathrm{OH}$ absorption to accurately examine the radial fluorescence signal distribution of the flame. The non-uniformity of laser sheet intensity distribution can be identified as the cause of the axial intensity distribution but this will be evaluated in Figs. 6 later in this section.

The averaged OH-PLIF images for the conditions $\beta=0.45$ and $P_{\mathrm{C}}=0.10,0.29$, and $0.49 \mathrm{MPa}$ are shown in Figs. 4(b) $\sim$ (d). A uniformed $\mathrm{OH}$ distribution was observed for $P_{\mathrm{C}}=0.10 \mathrm{MPa}$, as shown in Fig. 4(b), similar to Fig. 4(a). With $P_{\mathrm{C}}$ $=0.29$ and $0.49 \mathrm{MPa}$, the fluorescence intensity attenuation in the radial direction is due to the increase in the $\mathrm{OH}$ number density. The $\mathrm{OH}$ fluorescence intensity was observed to be slightly higher for $\phi=0.80$ than for $\phi=1.00$ in all $P_{\mathrm{C}}$ conditions. One-dimensional numerical simulation indicated that for $\phi=0.80$ and $\phi=1.00$ at $P_{\mathrm{C}}=0.10 \mathrm{MPa}$, the flame temperatures $10 \mathrm{~mm}$ downstream of flame surface were $2769 \mathrm{~K}$ and $2685 \mathrm{~K}$, respectively, and the $\mathrm{OH}$ mole fractions were 0.035 or 0.037 , respectively. The difference in the flame temperature is approximately $80 \mathrm{~K}$ while the difference in the mole fraction was approximately 0.002 . The results for $P_{\mathrm{C}}=0.29$ and $0.49 \mathrm{MPa}$ showed a similar trend. The fluctuation of $f_{\mathrm{B}} / T$ with the range of temperature differences was lower than $0.1 \%$. However, there are other spectroscopic parameters with temperature dependences, such as the quenching rate or fluorescence lifetime of excited molecule (Bergano et al., 1983). It was evident that the fluorescence signal intensity decreases with increasing temperature, and the decrease of the fluorescence signal intensity is more dominant than the increase of the OH mole fraction for $\phi=0.80$ and $\phi=1.00$. The relevance of the equivalence ratio variation will be discussed later in chapter 3.3.

In higher pressure conditions of $P_{\mathrm{C}}=0.29$ and $0.49 \mathrm{MPa}$, a disturbance in the outer burned gas surface began to be

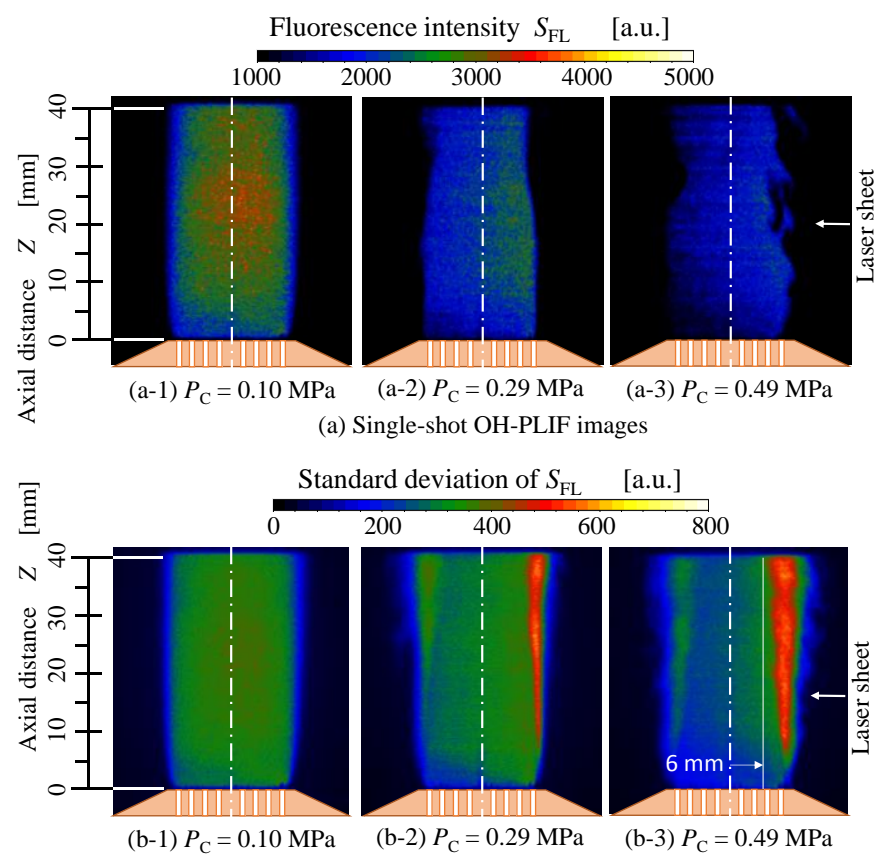

(b) Standard deviation distributions

Fig. 5 Images acquired at $P_{\mathrm{C}}=0.10$ to $0.49 \mathrm{MPa}, \beta=0.45$, and $\phi=1.00$. (a) Single-shot OH-PLIF images. (b) Standard deviation distribution. 


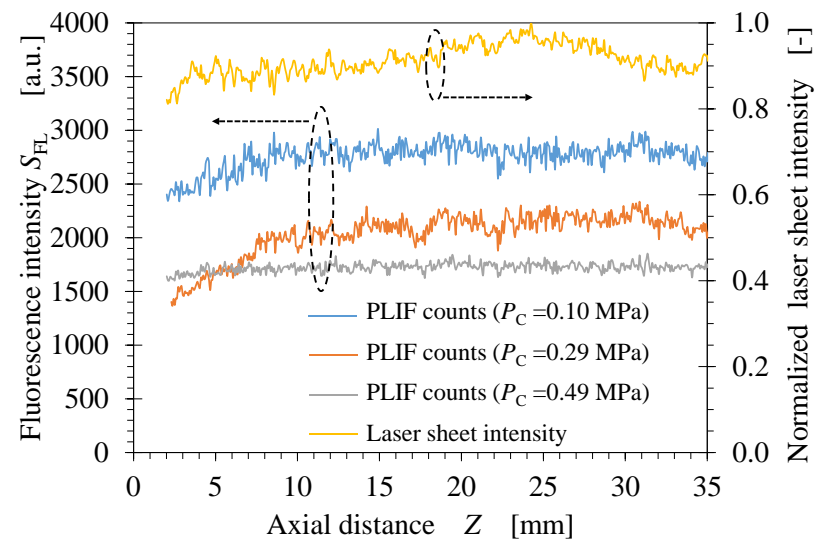

(a) Variation with $P_{\mathrm{C}}(\beta=0.45, \phi=1.0)$

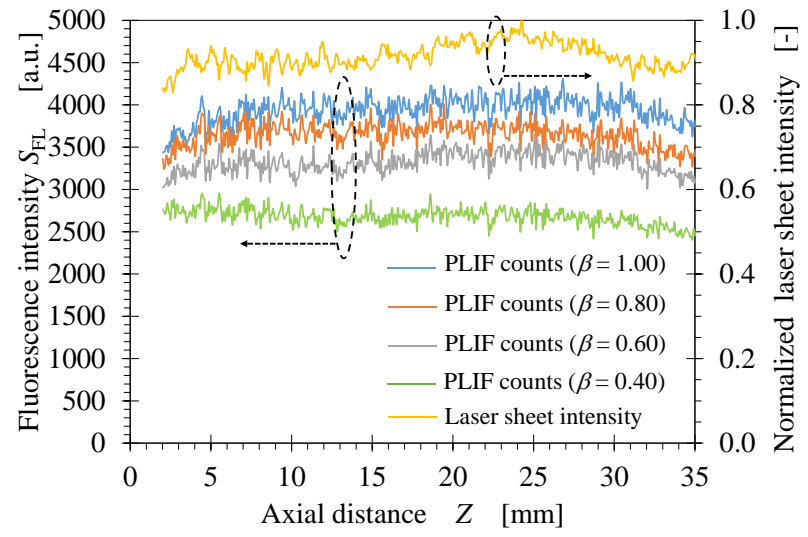

(b) Variation with $\beta\left(P_{\mathrm{C}}=0.10 \mathrm{MPa}, \phi=1.0\right)$

Fig. 6 Axial distributions of $\mathrm{OH}$ fluorescence intensity, $S_{\mathrm{FL}}$, corrected by laser sheet intensity. The normalized laser sheet intensity is also shown in these figures.

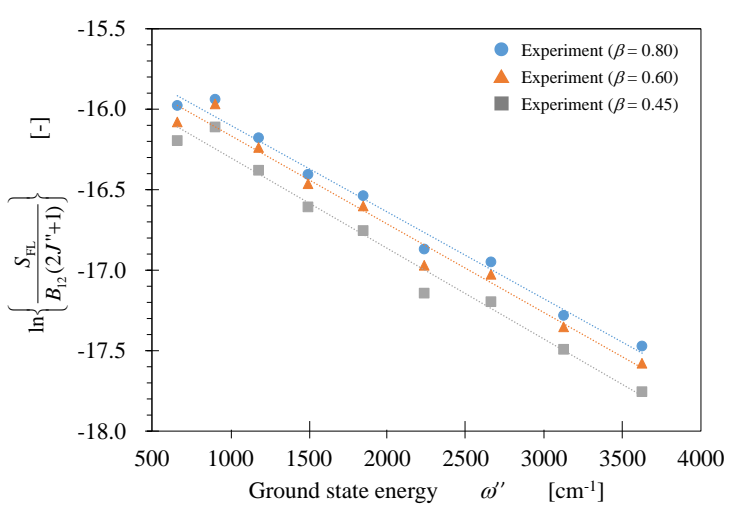

(a) Boltzmann-plot $(Z=10 \mathrm{~mm})$

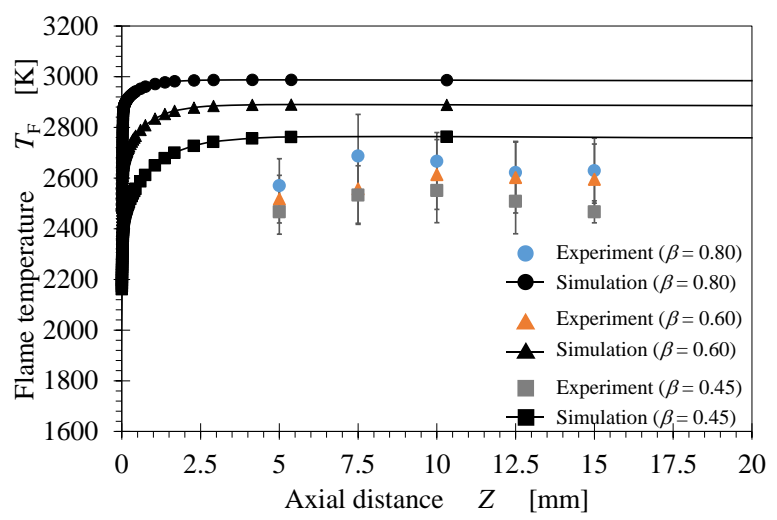

(b) Axial distributions of flame temperature

Fig. 7 Axial distributions of flame temperature for $P_{\mathrm{C}}=0.10 \mathrm{MPa}$ and $\phi=1.0$. (a) Boltzmann plot obtained at $Z=10 \mathrm{~mm}$. (b) Axial temperature distributions at $\beta=0.80,0.60$ and 0.55. In Fig. 7, the dashed lines represent the experimental results and the solid lines represent the 1-D flame simulation results.

observed where the burned gas and ambient air featured a large temperature gradient. To evaluate this disturbance, singleshot OH-PLIF images with $\beta=0.45$ and $\phi=1.0$ and the corresponding standard deviation distributions are shown in Fig. 5. The single shot OH-PLIF image was chosen randomly from the entire 100 images that were averaged in Fig. 4. As shown in Fig. 5, there were no disturbance in the outer surface of the burned gas at $P_{\mathrm{C}}=0.10 \mathrm{MPa}$, and the standard deviation distribution matched the $\mathrm{OH}$ intensity distribution reasonably well. It can therefore be concluded that when $P_{\mathrm{C}}$ $=0.10 \mathrm{MPa}$, the standard deviation under 400 in arbitrary unit to systematic factor such as shot noise of the ICCD. On the other hand, when $P_{\mathrm{C}}=0.29$ and $0.49 \mathrm{MPa}$, the disturbance in the surface of burned gas can be observed in the single shot image, and the standard deviation quantity is high in the corresponding regions. However, the region near the axial center that was within $6 \mathrm{~mm}$ of radial distance had a standard deviation quantity close to that of the atmospheric pressure condition. In conclusion, when considering the distributions of quantities in the burned gas, the region near the axial center should be chosen to eliminate the surface disturbance of burned gas.

The $\mathrm{OH}$ fluorescence intensity in the axial direction along the burner's central axial for $\phi=1.0$ at each $P_{\mathrm{C}}$ and $\beta$ is shown in Figs. 6. The laser sheet intensity distribution was acquired using a quartz cell enclosed with acetone that was used to correct the fluorescence intensity distribution. The acquired laser sheet intensity is also shown in Figs. 6. Due to the quartz cell shape, the axial distance was corrected to $Z=2 \sim 35 \mathrm{~mm}$. For each $P_{\mathrm{C}}$ and $\beta$ condition, the region of $Z=$ $7 \sim 35 \mathrm{~mm}$ region has a relatively uniform fluorescence intensity. In the $Z<7.0 \mathrm{~mm}$ region, the fluorescence intensity decreases due to the influence of heat loss to the nozzle surface. For $P_{\mathrm{C}}=0.49 \mathrm{MPa}$, there were no significant intensity decreases compared with the case of $P_{\mathrm{C}}=0.10$ and $0.29 \mathrm{MPa}$ due to the high heat release and thinning of the flame thickness.

The temperature distributions of the fuel in the radial direction at $P_{\mathrm{C}}=0.10 \mathrm{MPa}$ were evaluated by the temperature 
measurement results of the Boltzmann plot and one-dimensional simulation results as shown in Figs. 7. The equivalence ratio was constant at $\phi=1.0$ and oxygen-enrichment ratios of $\beta=0.80,0.60$, and 0.45 were evaluated. Figure 7(a) shows the Boltzmann plot taken at an axial distance of $Z=10 \mathrm{~mm}$. The slope was calculated using least-squares fitting for each $\beta$ condition. The results measured from $\mathrm{Q}_{1}(1)$ to $\mathrm{Q}_{1}(4)$ excitations were excluded in all the calculations, because the fluorescence intensities were much lower compared with higher $J$ " cases. Moreover, as $J$ " increases, the Boltzmann equilibrium by the rotational relaxation at the excited vibrational level is completed more certainly compared with the case of using lower $J$ " (Crosley, 1981). Thus, the results measured from $\mathrm{Q}_{1}(5)$ to $\mathrm{Q}_{1}(13)$ excitations were selected to calculate slops of the fitting lines for the Boltzmann-plots. Figure 7(b) shows the axial distribution of the flame temperature $T_{\mathrm{f}}$ at positions $Z=5,7.5,10,12.5$ and $15 \mathrm{~mm}$ calculated from the Boltzmann plot and the one-dimensional simulated $T_{\mathrm{f}}$ distribution. It can be observed that the flame temperature was relatively constant around $Z=7.5 \sim 15 \mathrm{~mm}$ from Fig. 7(b). The temperature distribution can be assumed to be uniform similar to the fluorescence intensity distribution along the axial direction shown in Fig. 6. However, the experimental $T_{f}$ was approximately $250 \mathrm{~K}$ lower than the one-dimensional simulated results which did not consider heat loss to the burner nozzle surface. In addition to heat loss, the half width of transmittance of the optical filter used for the fluorescence detection resulted in the temperature difference. The half width of the filter used in this study was $10 \mathrm{~nm}$ which is approximately two times larger than the half width of $\mathrm{OH}(2,1)$ band fluorescence. However, the acquiring fluorescence spectrum slightly changes by the difference of the excitation line $J$ '. When $J$ " is too small or too large, the filter is not able to transmit the fluorescence completely, because the filter transmittance is invariable. In other words, the detecting capability for the fluorescence will be variable in such case. This is a cause of underestimation of the flame temperature (Anderson et al., 1982). However, to prevent the strong $\mathrm{OH}$ chemiluminescence interference caused by high flame temperatures, a relatively narrow half width band pass filter was selected. Therefore, there was a possibility of the experimentally derived temperature being lower than the actual flame temperature in this study. Even though the experimental results differed from the numerical simulation results, when $P_{\mathrm{C}}=0.10 \mathrm{MPa}$ and $\beta=0.80$ the flame temperature was higher than $2650 \mathrm{~K}$ and even when $\beta=0.45$ the flame temperature was higher than $2450 \mathrm{~K}$ which is beyond temperature measurements by a thermocouple. It is rare to form such a high temperature flame for a calibration burner. The $\mathrm{OH}$ fluorescence intensity distribution at $Z=7 \sim 35 \mathrm{~mm}$ was uniform and the flame temperature was also uniform for a wide range of $Z=7.5 \sim 15 \mathrm{~mm}$. The calibration burner makes it possible to efficiently quantify each physical parameter not only in one-dimensional measurements but also in twodimensional measurement and withstands temperatures higher than $2650 \mathrm{~K}$. Thus, it can be concluded that the proposed calibration burner has met the requirements for a spectroscopic measuring calibration burner.

\subsection{Results of $\mathrm{OH}$ and $\mathrm{CH}$ chemiluminescence measurement}

Figure 8 shows the $\mathrm{OH}$ and $\mathrm{CH}$ chemiluminescence acquired by the experiments for $\beta=0.45$ at each pressure condition. The figure also shows the equivalence dependence of $\mathrm{OH}^{*}$ and $\mathrm{CH}^{*}$ partial pressures $X$ which corresponds to

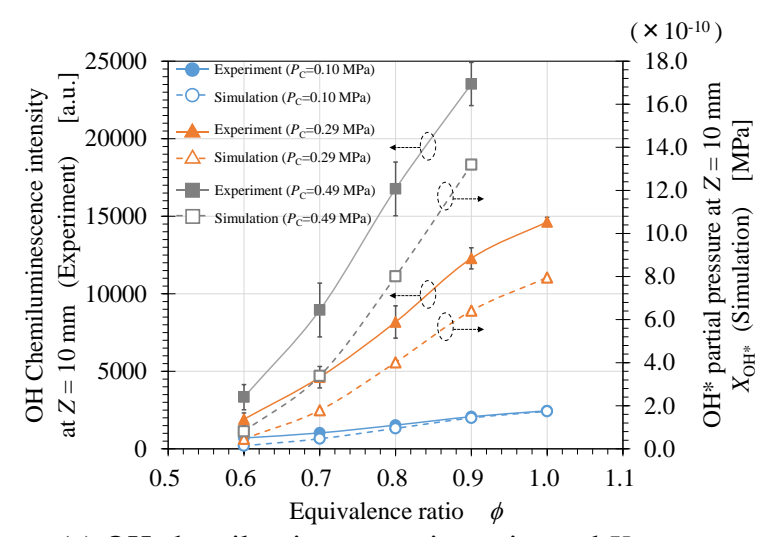

(a) $\mathrm{OH}$ chemiluminescence intensity and $X_{\mathrm{OH}^{*}}$

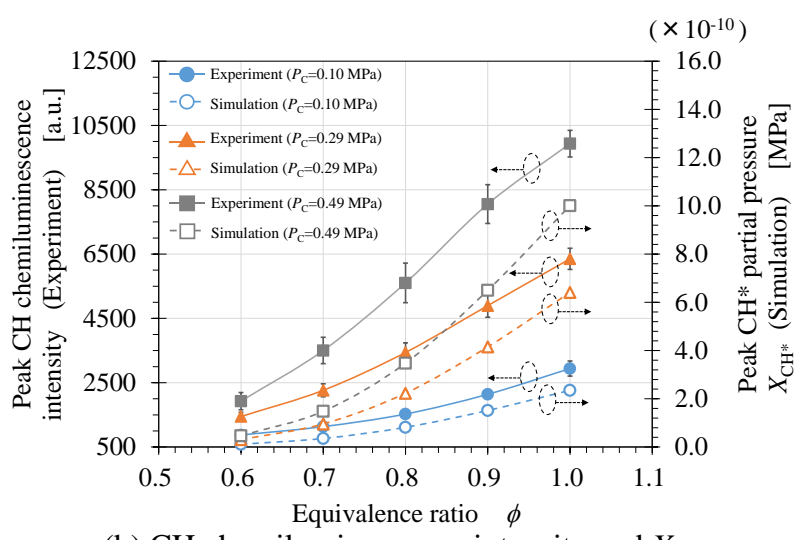

(b) $\mathrm{CH}$ chemiluminescence intensity and $X_{\mathrm{CH}^{*}}$

Fig. 8 Equivalence ratio dependence of acquired chemiluminescence intensity and simulated relative molar concentration of excited species. In Fig. 8, filled markers represent the experimental results and unfilled markers represent the simulated results. 


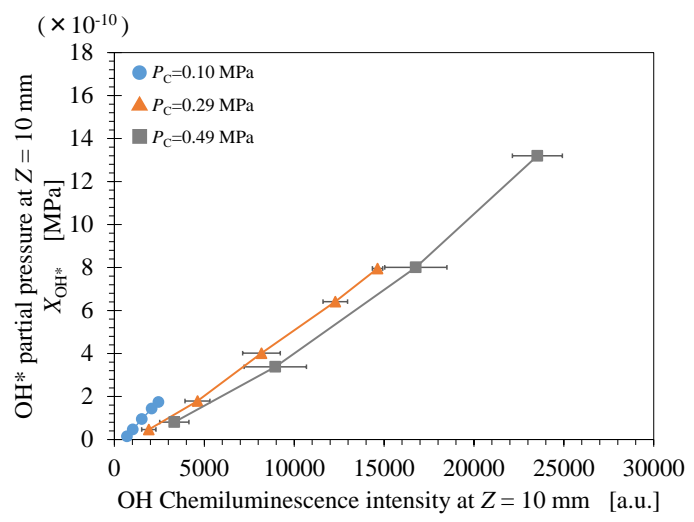

(a) OHchemiluminescence intensity and $X_{\mathrm{OH}^{*}}$

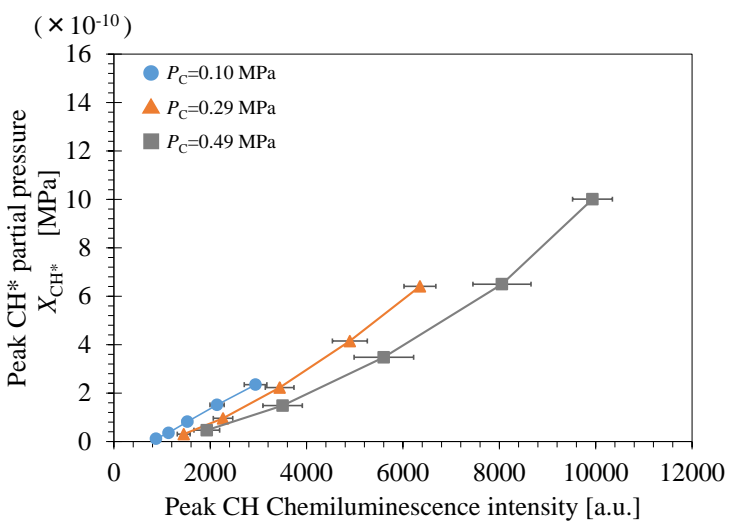

(b) CHchemiluminescence intensity and $X_{\mathrm{CH}^{*}}$

Fig. 9 Relationships between chemiluminescence and relative molar concentration for each $\phi$ and $P_{\mathrm{C}}$ condition.

the chemical species partial pressure derived from the one-dimensional simulation. Because an outer flame was formed with the fuel enriched conditions, the results for $\phi=0.60 \sim 1.0$ were examined. As mentioned above in chapter 3.2, position $Z=10 \mathrm{~mm}$ was selected for $X_{\mathrm{OH}^{*}}$ evaluation because the $\mathrm{OH}$ partial pressure and flame temperature in the axial direction can be regarded as uniform. Because $\mathrm{CH}$ only exists in the flame reaction zone, the peak intensity value near the nozzle surface was selected for $X_{\mathrm{CH}^{*}}$. The burner center was selected as the radial position for both $X_{\mathrm{OH}^{*}}$ and $X_{\mathrm{CH}^{*}}$ evaluation. As shown in Fig. 8, the equivalence ratio dependence of $\mathrm{OH}^{*}$ and $\mathrm{CH}^{*}$ partial pressures corresponded well with the chemiluminescence results. Thus, it can be concluded that the experimental flame temperature and the variation of the corresponding chemical specie partial pressure with equivalence ratio are reasonable. In Fig. 4, the OH fluorescence intensity of $\phi=0.80$ was originally thought to be higher than that of $\phi=1.0$ from the PLIF measurement due to the decrease of $\mathrm{OH}$ concentration; however, the relationship of chemiluminescence and $X_{\mathrm{OH}^{*}}$ indicate that the flame temperature or $\mathrm{OH}$ concentration was actually higher at $\phi=1.0$ than at $\phi=0.8$. This confirms that the fluorescence intensity decreases due to the increased flame temperature.

To verify whether the chemiluminescence intensity and partial pressures of each chemical species qualitatively corresponded, the chemiluminescence versus partial pressures were plotted, as shown in Fig. 9. It can be confirmed from Fig. 9(a) that the relationship between the $\mathrm{OH}$ chemiluminescence intensity and $X_{\mathrm{OH}^{*}}$ has a slight convex curvature but is relatively linear. This curvature indicates that the rate of change of the simulated partial pressure of $\mathrm{OH}^{*}$ was higher than that of experimentally acquired $\mathrm{OH}$ chemiluminescence intensity. In Fig. 9(b), it can be observed that relationship between the $\mathrm{CH}$ chemiluminescence intensity and $\mathrm{X}_{\mathrm{CH}^{*}}$ has a stronger convex curvature than $\mathrm{OH}$, because $\mathrm{CH}$ exists near the nozzle surface where the temperature is lower than that at downstream region due to the heat loss. Because the increase rate of the excited chemical species concentration rises exponentially, the difference in concentration between the experimental and numerical simulation results becomes larger than when the flame temperature increases at high pressures. Therefore, the heat loss of the burner surface must be considered in a similar way as a conventional calibration burner such as McKenna burner to calibrate the experimental results when targeting a chemical species located near the burner.

\section{Conclusions}

To develop a spectroscopic measurement calibration burner that can be used in pure oxygen or oxygen enriched conditions at high pressures, a new calibration burner which has a high heat resistance due to employ a water-cooled multi-hole nozzle was developed in this study. The basic performance of the burner (such as burner heat resistance and the flame characteristics) was evaluated based on both the experiments and numerical simulations. The following results were obtained.

1. At a chamber pressure $P_{\mathrm{C}}=0.10 \mathrm{MPa}$ and an oxygen enrichment $\beta=0.40 \sim 1.0$, the spectroscopic measurement calibration burner designed in this study can form a remarkably stable $\mathrm{CH}_{4} / \mathrm{O}_{2} / \mathrm{N}_{2}$ oxygen-enriched flame. Even with $P_{\mathrm{C}}=0.29$ and $0.49 \mathrm{MPa}$, when $\beta=0.45$, a stable flame can be formed in a wide range of equivalence ratios $\phi$ $=0.6 \sim 1.2$. 
2. The maximum inner temperature of the nozzle becomes higher than $380 \mathrm{~K}$ when $P_{\mathrm{C}}=0.48 \mathrm{MPa}$ and $\phi=1.0$, but it is well under the softening temperature of the oxygen-free copper used for the nozzle, which is designed to be $470 \mathrm{~K}$ at maximum, indicating that the burner can be used in higher heat load conditions.

3. OH-PLIF measurement results and the standard deviation at each pressure condition indicate there are disturbances in the outer surface of the burned gas at $P_{\mathrm{C}}=0.29$ and $0.49 \mathrm{MPa}$ but the effect of the disturbance is small in the region within $6 \mathrm{~mm}$ of the burner axial center. Moreover, the $\mathrm{OH}$ fluorescence intensity distribution is approximately uniform in a wide range of axial directions $Z=7 \sim 35 \mathrm{~mm}$.

4. The flame temperature are distributed approximately uniformly along the axial direction for $Z=7.5 \sim 15 \mathrm{~mm}$. This was derived from the temperature measurement result using a Boltzmann plot at $P_{\mathrm{C}}=0.10 \mathrm{MPa}$. The measured flame temperature was $250 \mathrm{~K}$ lower than the one-dimensional simulation which did not consider heat loss, but the flame temperature is still higher than $2450 \mathrm{~K}$ at $\beta=0.45$ and $2650 \mathrm{~K}$ at $\beta=0.80$.

5. The equivalence ratio dependence of $\mathrm{OH}(0,0)$ band and $\mathrm{CH}(0,0)$ band chemiluminescence relatively matched the equivalence ratio dependence of $\mathrm{OH}^{*}$ and $\mathrm{CH}^{*}$ partial pressure obtained by one-dimensional simulation.

\section{Acknowledgment}

This work was supported by the JSPS Grant-in-Aid for Challenging Exploratory Research Grant Number 15K14244 and the Division for Interdisciplinary Advanced Research and Education, Tohoku University.

\section{References}

Anderson, W.R., Decker, L.J. and Kotler, A.J., Temperature profile of a stoichiometric CH4/N2O flame from laser excited fluorescence measurements on OH, Combustion and flame, Vol.48 (1982), pp.163-176.

Barlow, R.S., Karpetis, A.N., Frank, J.H. and Chen, J.Y., Scalar profiles and NO formation in laminar opposed-flow partially premixed methane/air flames, Combustion and Flame, Vol. 127, No. 3 (2001), pp.2102-2118.

Bergano, N.S., Jaanimagi, P.A. and Salour, M.M., Picosecond laser-spectroscopy measurement of hydroxyl fluorescence lifetime in flames, Optics Letters, Vol. 8, No. 8 (1983), pp.443-445.

Bessler, W.G., Schulz, C., Lee, T., Jeffries, J.B. and Hanson, R.K., Strategies for laser-induced fluorescence detection of nitric oxide in hige-pressure flames.I. A-X(0,0) Excitation, Applied Optics, Vol. 41, No. 18 (2002), pp.3547-3557.

Burke, U., Somers, K.P., O’Toole, P., Zinner, C.M., Marquet, N., Bourque, G., Petersen, E.L., Metcalfe, W.K., Serinyel, Z. and Curran, H.J., An ignition delay and kinetic modeling study of methane, dimethyl ether, and their mixtures at high pressures, Combustion and flame, Vol. 162 (2015), pp.315-330.

CHEMKIN-Pro: a software package for the analysis of gas-phase chemical and plasma kinetics (online), available from <http://www.ansys.com/products/fluids/ansys-chemkin-pro> (Accessed on 13 May, 2017).

Cheng, T.S., Yuan, T., Lu, C.-C. and Chao, Y.-C., The application of spontaneous vibrational raman scattering for temperature measurements in high pressure laminar flames, Combustion Science and Technology, Vol. 174 (2002), pp.111-128.

Crosley, D.R., Collisional Effects on Laser-Induced Fluorescence Flame Measuremnts, Opt. Eng., Vol. 20 (1981), pp.511521.

Daily, J. W., Laser Induced Fluorescence Spectroscopy in Flames, Progress in Energy and Combustion Science, Vol. 23, No. 2 (1997), pp.133-199.

Kojima, J. and Nguyen, Q.V., Development of a high-pressure gaseous burner for calibrating optical diagnostic techniques, NASA Technical Report, TM2003-212738 (2003), pp.2-9.

LIFBASE: A free software program to chart the spectroscopy of diatomic molecules (online), available from <https://www.sri.com/engage/products-solutions/lifbase> (accessed on 13 May, 2017).

Niioka, T., Kono, M. and Sato, J., Foundation of Combustion Phenomenon, Ohmsha, Ltd. (2001), p.178(in Japanese).

Nishimura, M., Suyari, M., Suzuki, T. and Takagi, T., Studies of Fuel Savings and NOx Reduction in Oxygen Enriched Air Combustion (I)-Examination of Fuel Savings and Possibility of Low NOx Combustion-, Journal of the Japan Institute of Energy, Vol. 79, No. 4 (2000), pp.321-328 (in Japanese).

Prucker, S., Meier, W. and Stricker, W., A flat flame burner as calibration source for combustion research: Temperatures and species concentration of premixed $\mathrm{H}_{2}$ /Air flames, Review of Scientific Instruments, Vol. 65, No. 2908 (1994), 
pp.2908-2911.

Sakiyama, Y., Kurosawa, Y., Gomi, M. and Suzuki, K., PLIF imaging measurements of OH and temperature distribution in burning flow fields, Technical Report of National Aerospace Laboratory (1999), pp.3-4(in Japanese).

Shi, B., Kowari, T., Matsuda, S., Shimokuri, D. and Ishizuka, S., Effects of oxygen concentration in the oxidizer on the stability of rapidly mixed type tubular flame combustion, Journal of the Combustion Society of Japan, Vol. 54, No. 169 (2012), pp.193-200(in Japanese).

Takeishi, H., Hayashi, J., Kono, S., Arita, W., IINO, K. and Akamatsu, F., Characteristics of ammonia/N2/O2 laminar flame in oxygen-enriched air condition, Transactions of the JSME (in Japanese), Vol. 81, No. 824 (2015), DOI: 10.1299/transjsme.14-00423(in Japanese).

Takeuchi, K., Nunome, Y., Tomioka, S., Tomita, T., Kudo, T., Hayakawa, A. and Kobayashi, H., Application of OH(2,0) band excitation planar laser-induced fluorescence to high-pressure $\mathrm{H}_{2} / \mathrm{O}_{2}$ jet diffusion flames for rocket combustion, Transactions of the Japan Society for Aeronautical and Space Sciences, Vol. 60, No. 2 (2017), pp.116-123.

Weigand, P., Lückerath, R. and Meier, W., Documentation of flat premixed laminar CH4/Air standard flames, Institute of Combustion Technology, DLR, Digital documentation, available form < http://www.dlr.de/vt/en/desktopdefault.aspx/tabid-3065/4632_read-6696/> (Accessed on 13 May, 2017). 